\title{
Adipose stem cell-derived extracellular matrix represents a promising biomaterial by inducing spontaneous formation of prevascular-like structures by mvECs
}

\author{
Svenja Nellinger ${ }^{1}$ (D) | Isabelle Schmidt ${ }^{2}$ | Simon Heine ${ }^{1}$ | Ann-Cathrin Volz ${ }^{1}$ | \\ Petra J. Kluger ${ }^{2}$
}

${ }^{1}$ Reutlingen Research Institute, Reutlingen University, Reutlingen, Germany

${ }^{2}$ School of Applied Chemistry, Reutlingen University, Reutlingen, Germany

\section{Correspondence}

Petra J. Kluger, Reutlingen University, Applied Chemistry, Alteburgstr. 150, 72762

Reutlingen, Germany.

Email: petra.kluger@reutlingen-university.de

\section{Funding information}

Ministry of Science, Research and the Arts (Baden-Württemberg, Germany), Landesgraduiertenförderung, Program: Intelligent Process and Material Development in Biomateriomics (University Tuebingen and Reutlingen)

\begin{abstract}
Tissue constructs of physiologically relevant scale require a vascular system to maintain cell viability. However, in vitro vascularization of engineered tissues is still a major challenge. Successful approaches are based on a feeder layer (FL) to support vascularization. Here, we investigated whether the supporting effect on the self-assembled formation of prevascular-like structures by microvascular endothelial cells (mvECs) originates from the $\mathrm{FL}$ itself or from its extracellular matrix (ECM). Therefore, we compared the influence of ECM, either derived from adipose-derived stem cells (ASCs) or adipogenically differentiated ASCs, with the classical cell-based FL. All cell-derived ECM ( $c d E C M)$ substrates enabled mvEC growth with high viability. Prevascular-like structures were visualized by immunofluorescence staining of endothelial surface protein CD31 and could be observed on all CdECM and FL substrates but not on control substrate collagen I. On adipogenically differentiated ECM, longer and higher branched structures could be found compared with stem cell cdECM. An increased concentration of proangiogenic factors was found in cdECM substrates and FL approaches compared with controls. Finally, the expression of proteins associated with tube formation (E-selectin and thrombomodulin) was confirmed. These results highlight cdECM as promising biomaterial for adipose tissue engineering by inducing the spontaneous formation of prevascular-like structures by mvECs.
\end{abstract}

\section{KEYWORDS}

adipose-derived stem cells, biomaterials, extracellular matrix, prevascular-like structures, tissue engineering

\section{1 | INTRODUCTION}

Adipose tissue is a highly metabolic and vascularized tissue. In native tissue, a dense capillary network provides the supply of nutrients and inspiratory gases to the residing cells and removes their waste products. Since the diffusion limit of oxygen is $<200 \mu \mathrm{m}$ (Olive, Vikse, \&
Trotter, 1992; Thomlinson \& Gray, 1955), the centers of large tissue constructs experience necrosis and volume loss without a functional vascular network. Consequently, there is an urgent need for fast vascularization following implantation of adipose tissue implants to maintain tissue mass and viability. In addition, for the in vitro use of tissue constructs, for example, as a testing system, a stable functional

This is an open access article under the terms of the Creative Commons Attribution License, which permits use, distribution and reproduction in any medium, provided the original work is properly cited.

(C) 2020 The Authors. Biotechnology and Bioengineering Published by Wiley Periodicals LLC 
vascular system would be desirable to allow constructs of a larger size and to maintain comprehensive cell behavior. Furthermore, such vascularized tissue constructs would allow in vitro investigations regarding the development and therapy of vascular diseases.

The inclusion of a functional vascular system remains one of the biggest challenges in three-dimensional (3D) tissue engineering. To date, there are several strategies to vascularize engineered 3D tissue constructs, for example, functionalized scaffolds, perfusion bioreactors, cocultures, and in vivo approaches (Laschke \& Menger, 2016). Proangiogenic factors immobilized in the scaffold material were found to enhance vascularization (Laschke et al., 2008; Yoon, Chung, Lee, \& Park, 2006). For example, vascular endothelial growth factor (VEGF) and basic fibroblast growth factor (bFGF) are known to induce vessel formation and platelet-derived growth factor (PDGF) $\beta$ supports stabilization of the newly formed vessels (Gaengel, Genove, Armulik, \& Betsholtz, 2009). Different coculture systems, including monolayer or spheroid cultures, demonstrated spontaneous formation of vascular-like structures (Walser et al., 2013; Wenz, Tjoeng, Schneider, Kluger, \& Borchers, 2018). In particular, the coculture of endothelial cells (ECs) with adipose-derived stem cells (ASCs) showed a beneficial effect on prevascular-like structure formation (Verseijden et al., 2012; Volz, Hack, Atzinger, \& Kluger, 2018). Abovementioned techniques help to prevascularize a construct, but the complete vascularization is only achieved post implantation in vivo (Laschke, Strohe et al., 2009; Laschke, Vollmar, \& Menger, 2009). So far, there is no successful in vitro approach to create a physiological and functional vascular system, which ensures adequate stability and reproducibility. In most approaches, some type of feeder cells are used to support the formation of vascular-like structures by ECs. This living cellular part impedes a commercial application due to the difficulties in handling and storage. In contrast, lyophilized acellular biomaterials can be stored for long periods. Moreover, they evoke far fewer concerns regarding their application in regenerative medicine compared with the approaches including living cells. However, changes in structure and composition may occur during dehydration of natural materials. Thus, it has to be clarified if the processing of a biomaterial affects its ability to influence cellular behavior. To address this issue, next to the effect of the wet hydrogel-like form, the effect of the dehydrated materials on cellular behavior should be investigated.

A critical requirement for engineering tissue constructs is the use of a suitable scaffold that provides appropriate biological and physicochemical properties. The cell surrounding material also plays an important role in vascularization. There are several synthetic and natural scaffold materials used for vascularized tissue engineering approaches, for example, polylactic acid, polyethylene glycol, collagen, or hyaluronic acid. However, the extracellular matrix (ECM) as the natural environment of the cells in vivo represents the most physiological biomaterial. A variety of ECM-hybrid materials and pure decellularized ECM were investigated towards their ability to support stem cell differentiation and (neo)vascularization in vivo and in vitro (Adam Young, Bajaj, \& Christman, 2014; Badylak, Freytes, \& Gilbert, 2009; Flynn, 2010; Flynn, Prestwich, Semple, \& Woodhouse, 2009). All these studies were performed with decellularized ECM derived from native tissue. For the past years, another source of natural ECM moves to the fore. In vitro generated cell-derived ECM (cdECM) was isolated from different cell-types (e.g., fibroblasts and ASCs) and used as a biomaterial in a variety of applications (Lu, Hoshiba, Kawazoe, \& Chen, 2011; Lu, Hoshiba, Kawazoe, Koda et al., 2011; Sart et al., 2016; Schenke-Layland et al., 2009; Wolchok \& Tresco, 2010). Several studies show that cdECMs, obtained from different cell-types, can induce adipogenic, chondrogenic, and osteogenic differentiation of ASCs indicating its influence on cell fate (Dzobo et al., 2016; Guneta et al., 2017; Guneta, Loh, \& Choong, 2016; Guo et al., 2013).

Our previous study revealed a spontaneous formation of prevascular-like structures by microvascular endothelial cells (mvECs) in a coculture with adipogenically differentiated ASCs (Volz et al., 2018). In the following the term "prevascular-like structures" defines the aggregation/alignment of CD31-positive mvECs to fiber- or network-like structures, which stand out from the rest of the cellular monolayer. This term was previously used by Verseijden, Posthumus-van Sluijs, Farrell et al. (2010) and Verseijden, Posthumus-van Sluijs, Pavljasevic et al. (2010) to describe the alignment of ECs in spheroids without lumen formation. The formation of vacuoles within ECs and subsequent lumenogenesis (tube formation) of prevascular-like structures requires the activation of cellular pathways and the transcription of different genes (Bayless \& Davis, 2002). Furthermore, the expression of E-selectin and thrombomodulin was shown to contribute to tube formation (Oh et al., 2007; Pan et al., 2017). In this study, we aimed to analyze whether the formation of prevascular-like structures by mvECs has to be attributed to cell-cell or cell-matrix interactions. The maintenance of the biological impact after processing and storage represents an important feature regarding the commercial application of biomaterials. The most common processing method for the preservation of biomaterials is drying. Consequently, we directly compared the effect of the hydrogel-like, wet cdECM and the dried $\mathrm{cdECM}$ as a coating regarding its ability to support the formation of prevascular-like structures by mvECs. The formation of vascular structures is rather a developmental process than a maintenance phenomenon as it can, for example, be found during (adipogenic) differentiation rather than in the stem cell niche. Thus, we tested whether there is a difference between cdECM derived from stem cells and adipogenically differentiated cells regarding their capability to induce prevascular-like structure formation.

\section{MATERIALS AND METHODS}

All research was carried out in accordance with the rules for the investigation of human subjects as defined in the Declaration of Helsinki. Patients provided written agreement in compliance with the Landesärztekammer Baden-Württemberg (F-2012- 078, for normal skin from elective surgeries).

\section{1 | Cell isolation and expansion}

ASCs were isolated from human tissue samples obtained from patients undergoing plastic surgery (Dr. Ziegler; Klinik Charlottenhaus, 
Stuttgart, Germany) as described before (Huber, Borchers, Tovar, \& Kluger, 2016). ASCs were initially seeded at a density of $5 \times 10^{3}$ cells/ $\mathrm{cm}^{2}$ in serum-free mesenchymal stem cell (MSC) growth medium (MSCGM, PELOBiotech) containing $5 \%$ human platelet lysate. ASCs were used up to passage three.

MvECs were isolated from juvenile foreskins (Dr. Yurttas, Stuttgart, Germany) as described before (Volz, Huber, Schwandt, \& Kluger, 2017). Briefly, dermis was cut into small pieces and digested in a dispase solution $(2 \mathrm{U} / \mathrm{ml}$; Serva Electrophoresis, Germany) overnight at $4^{\circ} \mathrm{C}$. After the removal of the epidermis, mvECs were isolated from the dermal layer by incubation with $0.05 \%$ trypsin in ethylenediaminetetraacetic acid (EDTA; Life Technologies, Germany) for $40 \mathrm{~min}$ at $37^{\circ} \mathrm{C}$ and mechanically isolated in mvEC growth medium-2 (EGM-2mv; Lonza, Switzerland). For cell expansion, mvECs were seeded with $5 \times 10^{3}$ cells $/ \mathrm{cm}^{2}$. MvECs were used up to passage three.

\section{2 | Generation of cell-derived extracellular matrix substrates and ASC feeder layer}

ASCs were seeded into 8-well chamber slides (ibidi, Germany) and 24-well plates, respectively at a density of $25 \times 10^{3}$ cells $/ \mathrm{cm}^{2}$ in serum-free MSCGM containing $5 \%$ human platelet lysate. At confluency, medium was changed to either serum-containing GM (Dulbecco's Modified Eagle's Medium (DMEM) with 10\% fetal calf serum
$(F C S)=s c d E C M)$ or adipogenic differentiation medium (DMEM with $10 \% \mathrm{FCS}, 1 \mu \mathrm{g} / \mathrm{ml}$ insulin, $1 \mu \mathrm{M}$ dexamethasone, $100 \mu \mathrm{M}$ indomethacin, $500 \mu \mathrm{M}$ 3-isobutyl-1-methylxanthine = acdECM) both supplemented with $50 \mathrm{\mu g} / \mathrm{ml} \mathrm{Na-L-ascorbate}$ and $1 \%$ penicillin/ streptomycin. The medium was changed every other day. At Day 7 , cells were lysed using hypotonic ammonium hydroxide solution and ECM was washed with ultrapure water. For dry ECM approaches (=dry), ECM was dried at room temperature (RT) and for wet ECM approaches (=wet), ECM was stored in ultrapure water at $4^{\circ} \mathrm{C}$ until seeded with mvECs. Cellular substrates (feeder layer [FL]) were seeded with mvECs without lysis of ASCs (Figure 1).

\section{3 | Macroscopic pictures and degree of swelling}

Macroscopic pictures of wet cdECM substrates were taken directly after cell removal. To investigate the water uptake and to calculate the degree of swelling, lyophilized cdECMs were weighed to determine the dry weight (weight [dry cdECM]). Subsequently, cdECMs were swollen in demineralized water for $24 \mathrm{hr}$ at RT and weighed again (weight [swollen cdECM]).

The degree of swelling was calculated as

$$
\begin{aligned}
& \text { Degree of swelling [\%] } \\
& =\frac{\text { weight }(\text { swollen cdECM })-\text { weight }(\text { dry cdECM })}{\text { weight }(\text { dry cdECM })} \times 100 .
\end{aligned}
$$

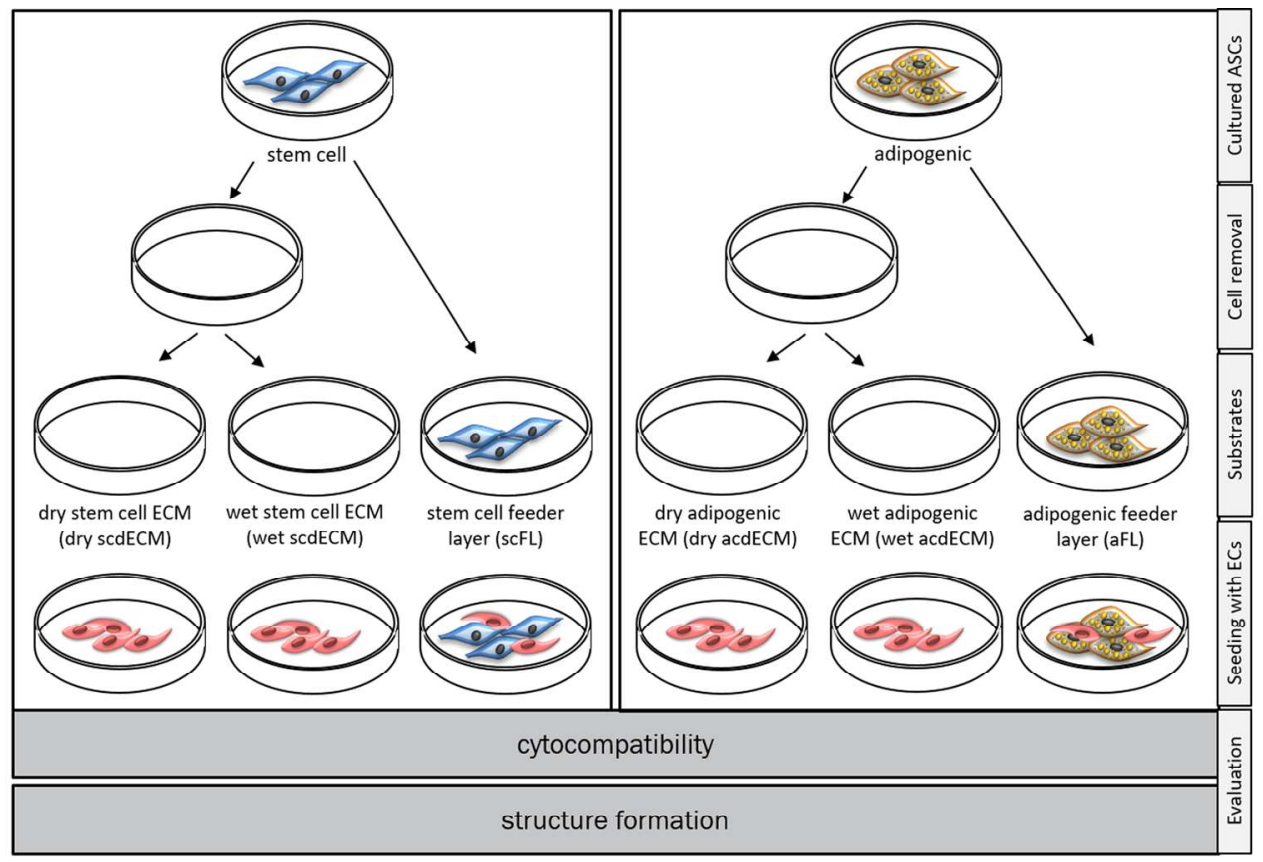

FIGURE 1 Schematic overview of the study procedure. The proangiogenic potential of different acellular and cellular substrates was analyzed. ASCs were cultured in growth and adipogenic differentiation medium respectively for 7 days. For acellular ECM substrates (cdECM), ASCs were removed and the remaining $\mathrm{CdECM}$ was dried or stored under wet conditions. For cellular substrates (FL), ASCs were not removed. MvECs were seeded onto the different substrates. Cytocompatibility was determined at Day 3 and prevascular-like structure formation was determined at Day 14 of cell culture. ASCs, adipose-derived stem cells; cdECM, cell-derived extracellular matrix; FL, feeder layer; MvECs, microvascular endothelial cells [Color figure can be viewed at wileyonlinelibrary.com] 


\subsection{Immunofluorescence staining of fibronectin and quantification of pore size}

For immunofluorescence (IF) staining of fibronectin, cdECM substrates were fixed in 4\% paraformaldehyde (Carl Roth, Germany) for $10 \mathrm{~min}$ followed by incubation with blocking solution, consisting of $3 \%$ bovine serum albumin (BSA; Biomol, Germany) in $0.1 \%$ Triton $X$ (Sigma Aldrich, Germany) for 30 min to block unspecific binding sites. Subsequently, the primary antibody (mouse anti-fibronectin, Santa Cruz, Germany; 1:200) was incubated for $1 \mathrm{hr}$ at RT. After washing three times with $0.1 \%$ Tween-20 (Sigma Aldrich, Germany) in phosphate-buffered saline (PBS), secondary antibody (anti-mouse Cy3, Dianova, Germany; 1:250) was incubated for $30 \mathrm{~min}$ at RT. Both were diluted in blocking solution. Images were taken with Axio Observer microscope and Axiocam 305 color using ZENblue software (Carl Zeiss, Germany). The pore size of the different cdECM substrates was quantified using ImageJ based on the IF images. The 100 largest pores for each image were determined and results are given as the mean.

\subsection{Seeding of mvECs on cell-derived ECM and feeder layer}

Isolated dry and wet cdECM substrates were reseeded with mvECs at a density of $1 \times 10^{4}$ cells $/ \mathrm{cm}^{2}$ in a defined mvEC adipocyte coculture medium (Volz et al., 2018). For FL approaches, mvECs were directly seeded on top of adipogenically differentiated and undifferentiated ASCs at a density of $1 \times 10^{4}$ cells $/ \mathrm{cm}^{2}$ in defined coculture medium, developed by us earlier (Volz et al., 2018). Cells were cultured for 14 days and the medium was changed every other day (Figure 1). As a control, all experiments were performed on Collagen I (COL I; rat tail; $250 \mu \mathrm{g} / \mathrm{ml}$ in $0.1 \%$ acetic acid) coated tissue culture polystyrene (COL I) and uncoated tissue culture polystyrene (TC). All media were supplemented with $1 \%$ penicillin/streptomycin.

\section{6 | Cytocompatibility}

Cytocompatibility of the cdECM substrates was demonstrated by the analysis of lactate dehydrogenase (LDH) in the cell culture supernatant. At Day 3 after seeding, an LDH assay (TaKaRa Bio Europe, France) was performed according to the manufacturer's instructions. To exclude the remaining LDH from cell lysis, LDH concentration from supernatant from cdECM substrates without mvECs was determined. Values were subtracted from the LDH concentrations measured from mvECs on the different cdECM substrates. On Day 14 , live-dead staining was performed to assess the viability of cultured cells. Before staining the cells were washed in PBS (Biochrom, Germany) and subsequently treated with staining solution, consisting of $200 \mathrm{ng} / \mathrm{ml}$ fluorescein diacetate (FDA, Sigma Aldrich, Germany) and $20 \mu \mathrm{g} / \mathrm{ml}$ propidium iodide (PI, Sigma Aldrich, Germany) in DMEM, for $15 \mathrm{~min}$ at $37^{\circ} \mathrm{C}$. Finally, cells were imaged in PBS with calcium and magnesium at RT with the Axio Observer microscope and Axiocam 305 color camera using ZENblue software (Carl Zeiss, Germany). The number of dead and viable cells was quantified using the software ImageJ and results were depicted as a percentage.

\subsection{Immunofluorescence staining of cell-specific proteins}

For IF staining of cell-specific proteins, cells were fixed in 4\% paraformaldehyde for $10 \mathrm{~min}$ and permeabilized for $10 \mathrm{~min}$ with $0.1 \%$ Triton $\mathrm{X}$ in PBS. Following, cells were incubated in blocking solution, consisting of $3 \% \mathrm{BSA}$ in $0.1 \%$ Triton $\mathrm{X}$ for $30 \mathrm{~min}$ to block unspecific binding sites. Primary antibodies (mouse anti-CD31, 1:50, Dako, Germany; rabbit anti-CD31, 1:200, abcam, GB; goat anti-E-selectin, 1:200, R\&D Systems; sheep anti-thrombomodulin, 1:200, R\&D Systems) were diluted in blocking solution and incubated with samples for $2 \mathrm{hr}$ at RT. Secondary antibodies (antirabbit Alexa FluorTM 488, abcam, GB; antimouse Cy3, Dianova, Germany; donkey anti-sheep Alexa FluorTM 647, abcam, GB; donkey anti-goat Alexa FluorTM 594, abcam, GB) were diluted 1:250 in blocking solution and incubated with samples for $30 \mathrm{~min}$ at RT.

\subsection{Enzyme-linked immunosorbent assay}

For characterization of cdECM substrates regarding growth factors composition, substrates were washed 3 days in culture medium. For the characterization of $\mathrm{FL}$, medium from Day 3 was collected. Quantification of growth factors VEGF, bFGF, and PDGF $\beta$ was performed using enzyme-linked immunosorbent assays (ELISA) (all PEPROTech, Germany) according to the manufacturer's instructions. The converted 3,3',5,5'-tetramethylbenzidin was read out at $450 \mathrm{~nm}$ with a wavelength correction set at $620 \mathrm{~nm}$ (TECAN Saphire II, Tecan, Switzerland).

\section{9 | Statistical analysis}

All experiments were performed at least three times, using cells from at least three different biological donors of ECs. The obtained data were compared by a one-way analysis of variance with repetitive measurement and a Bonferroni post hoc test using OriginPro 2018b. Statistical significances were stated as ${ }^{*} p \leq .05$, very significant as ${ }^{* *} p \leq .01$, and highly significant as ${ }^{* * *} p \leq .001$.

\section{3 | RESULTS}

\subsection{Macroscopic pictures and degree of swelling}

Macroscopic pictures showed that wet scdECM and acdECM substrates exhibited a transparent gel-like appearance on the bottom of 
a petri dish (Figure 2). Determination of the degree of swelling of the different $\mathrm{cdECM}$ substrates revealed a higher water uptake capacity of $\operatorname{acdECM~}(2,357.6[ \pm 201.1] \%)$ compared with the $\operatorname{scdECM}(1,624.3$ $( \pm 96.4) \%)$. Quantification of the pore size in the IF staining of fibronectin revealed smaller pores in the ScdECM substrates compared with the acdECM substrates.

\subsection{Acellular and cellular substrates are cytocompatible for mvECs}

Cytocompatibility of the materials was determined by the measurement of the release of LDH after seeding with mvECs. LDH is an enzyme that is released during cell death and therefore can be used to quantify cytotoxicity. LDH release by mvECs seeded on the different substrates was measured 3 days after seeding (Figure 3a). The values of TC were set as $100( \pm 3.5) \%$. For cdECM substrates, values were normalized to TC. Results showed no significant increase of released LDH of mvECs when seeded on COL I coating (89.4 [ \pm 13.8$] \%)$, dry scdECM (113.7 [ \pm 31.0$] \%)$; dry $\operatorname{acdECM}(108.0[ \pm 29.0] \%)$, wet $\operatorname{scdECM}(96.3[ \pm 33.4] \%)$, or wet $\operatorname{acdECM}(93.4[ \pm 29.0] \%)$. For the stem cell and adipogenic FL substrates, values were normalized to stem cell $\mathrm{FL}$ approach without mvECs (FL stem cell), which was set as $100( \pm 3.3) \%$. For adipogenic FL (FL adipogenic: $157.9[ \pm 13.4] \%$ ) approach, a higher $\mathrm{LDH}$ release was found compared to stem cell FL. As in the cdECM approaches, no significant increase in LDH release was observed when mvECs were seeded onto the FL for stem cell and adipogenically differentiated cells (FL stem + mvECs: $126.1[ \pm 15.8] \%$; FL ad + mvECs: $176.8[ \pm 25.0] \%)$.
The viability of mvECs cultured on the different substrates was assessed on Day 14 after seeding with mvECs by live-dead staining with FDA and PI (Figure 3b). Results showed that mvECs were viable on all acellular and FL substrates on Day 14 and only a few dead cells could be found. Quantification of the percentage of dead cells revealed $<1 \%$ of dead cells on all acellular substrates (TC: 0.14 [ \pm 0.08$] \%$; COL I: $0.29[ \pm 0.21] \%$; dry scdECM: $0.46[ \pm 0.11] \%$; dry acdECM: $0.61[ \pm 0.13] \%$; wet scdECM: $0.25[ \pm 0.23] \%$; and wet acdECM: $0.45[ \pm 0.26] \%)$. On FL substrates higher amounts of dead cells were found in adipogenic approaches compared to the stem cell approach (FL stem cell: $0.25[ \pm 0.08] \%$; FL adipogenic: $4.58[ \pm 0.19] \%$; FL stem cell + mvECs: $0.37 \quad[ \pm 0.18] \% ; \quad F L$ adipogenic + mvECs: $3.25[ \pm 1.66] \%)$.

\section{3 | Cell-derived ECM substrates support the formation of prevascular-like structures by mvECs}

To investigate the effect of cdECM substrates on the formation of prevascular-like structures by mvECs, CD31 was visualized by IF staining (Figure 4). CD31 is a specific endothelial surface protein mainly localized on cell-cell connections and mainly responsible for the control of leukocyte transmigration in vivo (Piali et al., 1995). The staining pattern revealed that mvECs grew to a confluent cell layer on all acellular substrates. The degree of structure formation on the different substrates was analyzed and quantified using ImageJ on basis of the CD31 IF images. The formation of prevascular-like structures by mvECs was detected on all tested substrates in contrast to the controls (TC and COL I) on which no structure formation was observed. Higher magnification of the cellular monolayer or the
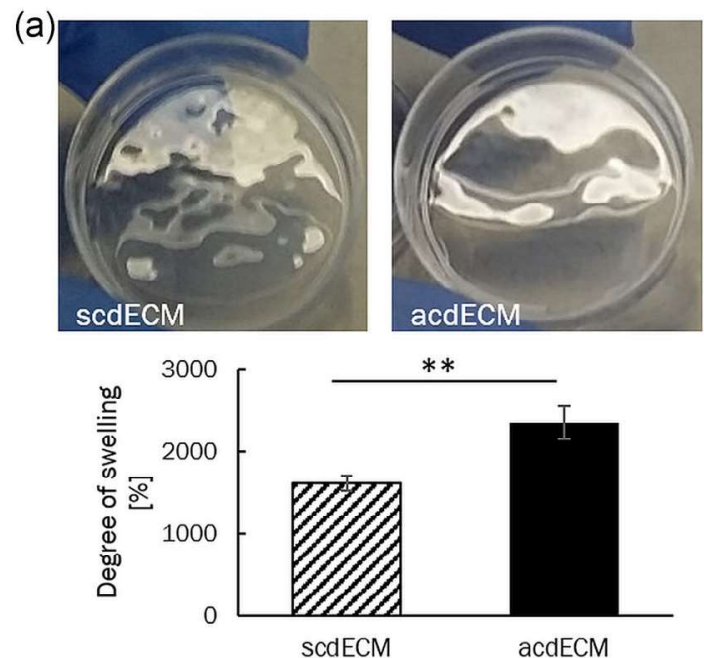

(b)
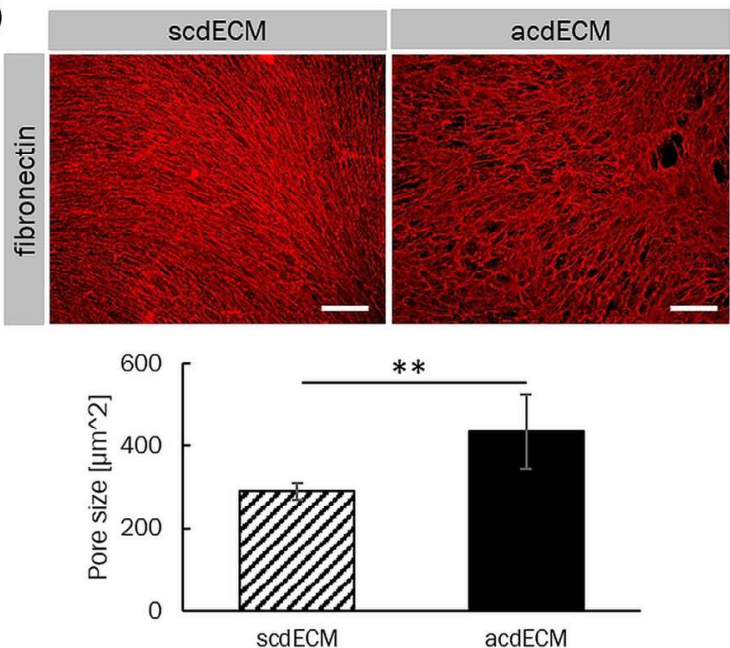

FIGURE 2 Degree of swelling of ECM substrates and staining of fibronectin and quantification of pore size. (a) Macroscopic pictures of cdECM substrates show a transparent gel-like cdECM on the bottom of the petri dish. The degree of swelling was calculated in percent. Results revealed a higher swelling rate of acdECM compared with the scdECM (diameter of the petri dish is $35 \mathrm{~mm}$ ). (b) Fixed cdECM samples were stained for fibronectin. Quantification of the pore sizes in fibronectin staining revealed smaller pores in the scdECM substrate compared with the acdECM substrate (fibronectin indicated in red; scale bar $=200 \mu \mathrm{m}$ ). AcdECM, adipogenic cell-derived extracellular matrix; scdECM, stem cell-derived extracellular matrix. $\left({ }^{* *} p \leq .01\right)$ [Color figure can be viewed at wileyonlinelibrary.com] 
(a)

(b)
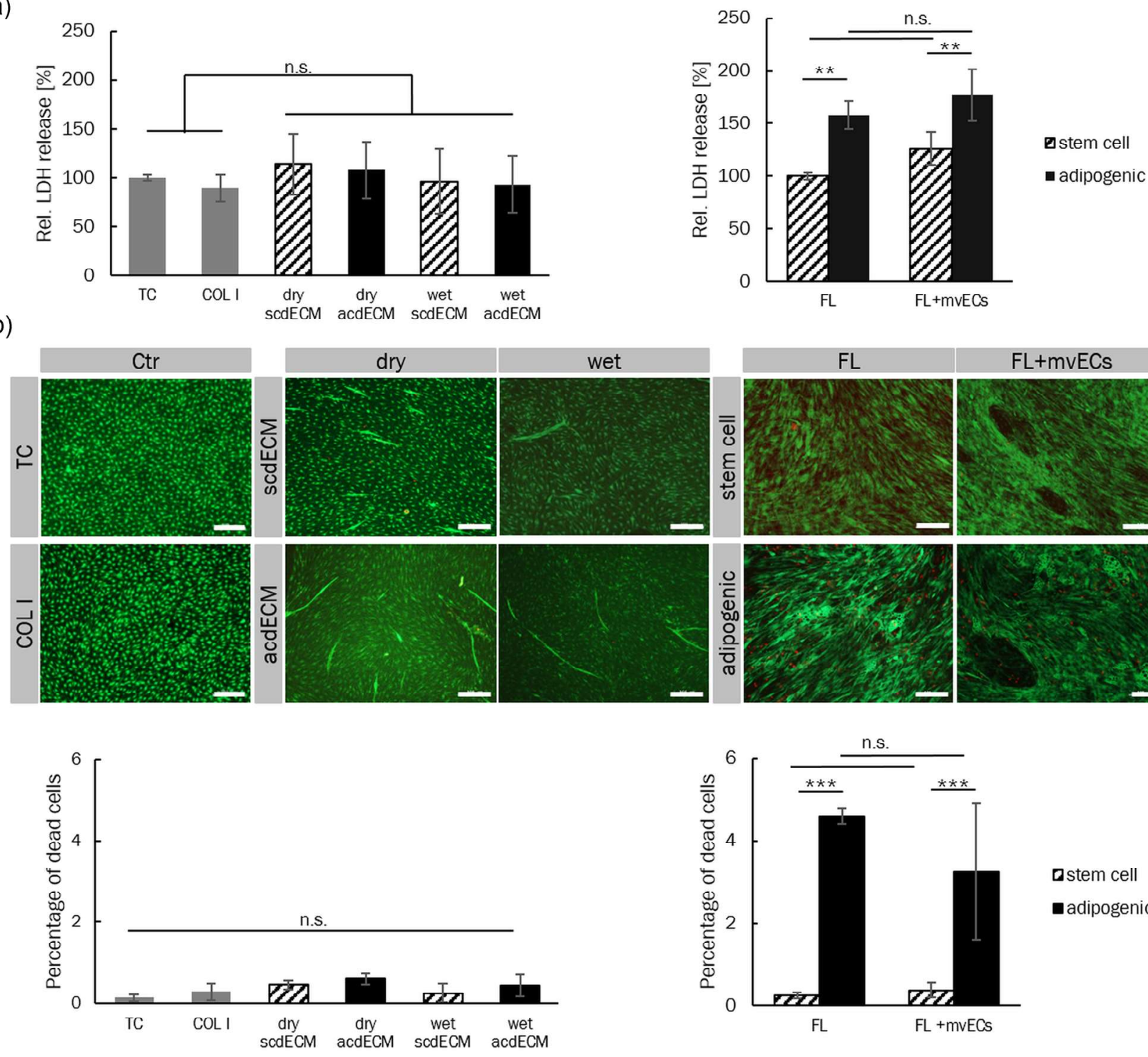

wet
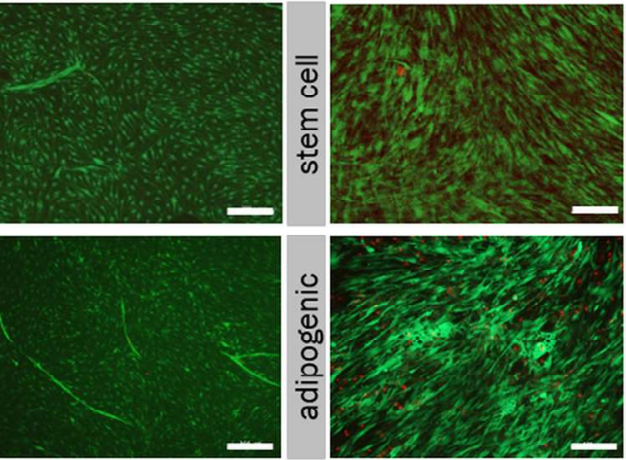

\section{$\mathrm{FL}+$ mvECs}



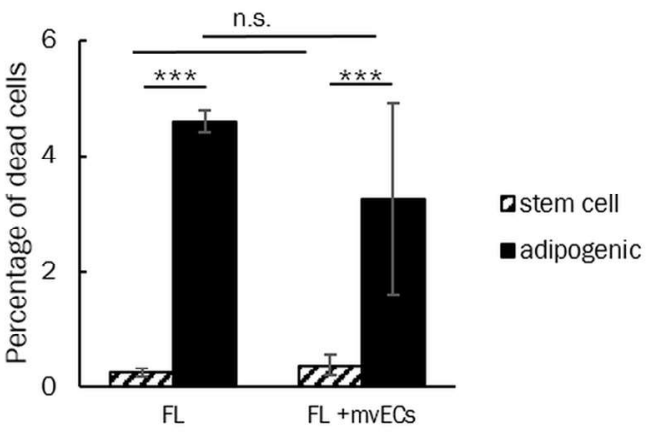

FIGURE 3 Cytocompatibility of the acellular and feeder layer substrates. About $1 \times 10^{4} \mathrm{cells} / \mathrm{cm}^{2} \mathrm{mvECs}$ were seeded in a defined medium onto the different substrates. (a) Relative LDH release was measured at Day 3 after seeding with mvECs. For acellular substrates, values were normalized to TC. No significant increase in LDH release could be observed on COL I coating or dry and wet cdECM for both, scdECM and acdECM. For FL approaches, values were normalized to stem cell FL without mvECs (FL stem cell). None of the FL approaches (stem cell and adipogenic differentiated) exhibited a significant increase of released LDH after seeding of mvECs (FL + mvECs). (b) Live-dead staining (FDA, indicating alive cells displayed in green/PI, indicating dead cells, displayed in red) was performed on Day 14 after seeding with mvECs. A confluent layer of viable cells was observed in all approaches. For each approach, the percentage of dead cells was quantified using ImageJ. The analysis revealed an amount of $<1 \%$ of dead cells for all acellular substrates with no significant differences between the different substrates. In FL approaches, a higher number of dead cells could be found in adipogenic approaches compared to stem cell approaches. Scale bar represents $200 \mu \mathrm{m}$. AcdECM, adipogenic cell-derived extracellular matrix; cdECM, cell-derived extracellular matrix; COL I, collagen I; FDA, fluorescein diacetate; FL, feeder layer; LDH, lactate dehydrogenase; mvECs, microvascular endothelial cells; ns, not significant; PI, propidium iodide; scdECM, stem cell-derived extracellular matrix; TC, tissue culture. $\left({ }^{* *} p \leq .01,{ }^{* * *} p \leq .001\right)$ [Color figure can be viewed at wileyonlinelibrary.com]

prevascular-like structures shows the typical localization of CD31 at cell-cell contacts. Quantification of the structures' lengths revealed longer structures in wet acdECM $(433.5( \pm 293.1) \mu \mathrm{m})$ substrate compared with both scdECM substrates (dry: $235.9( \pm 100.0) \mu \mathrm{m}$ and wet: $232.9( \pm 183.8) \mu \mathrm{m})$. Dry acdECM $(297.2( \pm 149.1) \mu \mathrm{m})$ substrates exhibited a slightly but not significantly higher structure length compared with dry and wet scdECM substrates. The lengths of prevascular-like structures found on the adipogenic FL (FL adipogenic: $483.5( \pm 287.4) \mu \mathrm{m})$ were significantly longer than those of all other approaches except for wet acdECM substrates. Prevascularlike structures on stem cell FL exhibited an average length per structure of $302.1( \pm 168.7) \mu \mathrm{m}$. Another essential criterion for the maturation of a vascular network is the formation of nodes. Therefore, the number of nodes formed by the mvECs on the different 

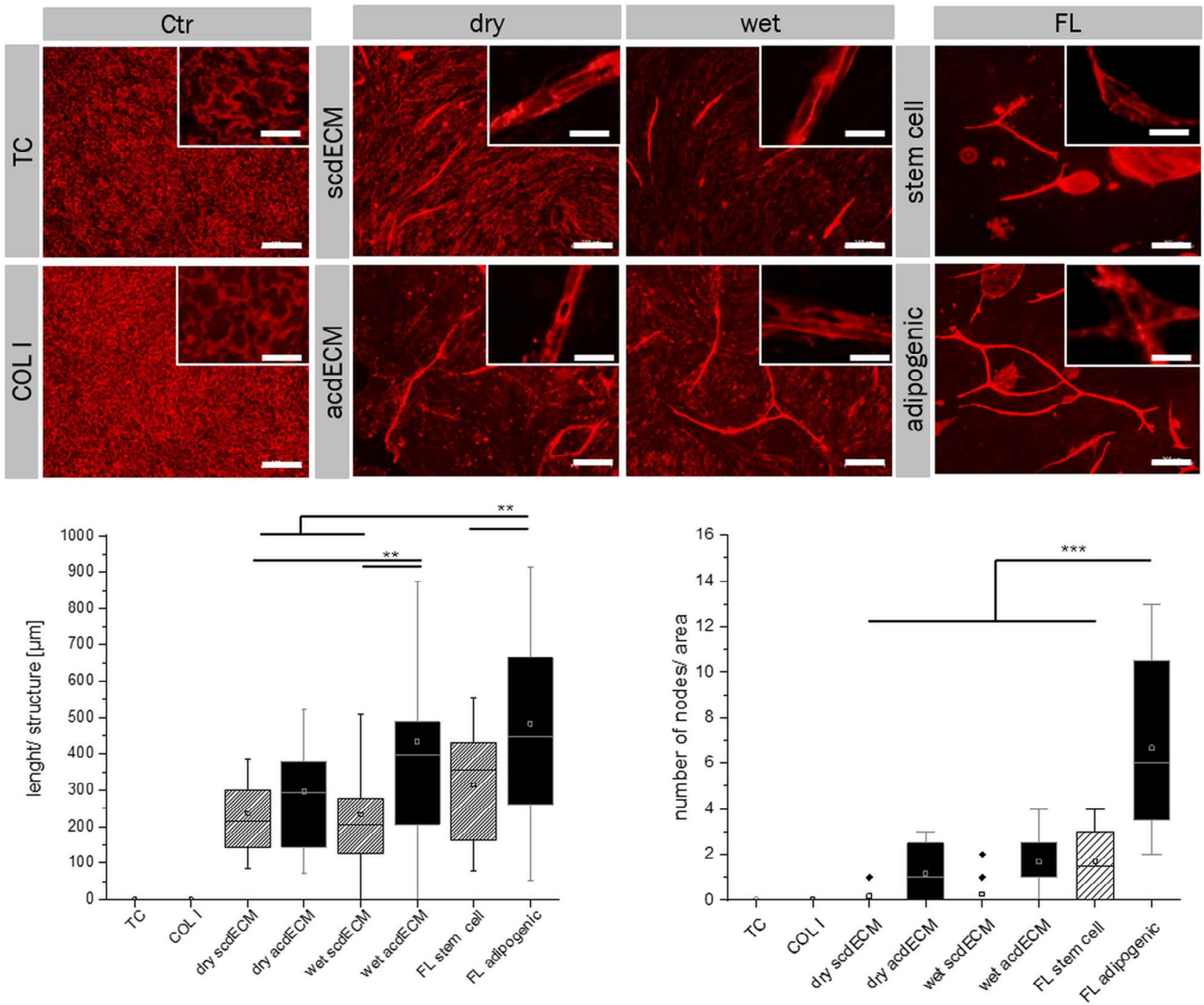

FIGURE 4 Formation of prevascular-like structures by mvECs on cellular and acellular ECM substrates. About $1 \times 10^{4} \mathrm{mvECs} / \mathrm{cm}^{2}$ were seeded in defined coculture medium onto the different substrates and were cultured for 14 days. Medium was changed three times a week. For determination of newly formed prevascular-like structures, IF staining of CD31 (indicated in red) was performed at Day 14 after seeding with mvECs. On controls ( $T C$ and COL I), a confluent layer of mvECs could be observed without any structure formation. On cdECM substrates, the formation of prevascular-like structures could be observed with the strongest manifestation on wet acdECM. The highest degree of structure formation could be observed on adipogenic FL. On stem cell FL cluster formation of mvECs could be found and a considerably lower degree of structure formation compared to the adipogenic approach was detected. For each representative overview image, a magnified section of the cellular monolayer or the prevascular-like structures is pictured in the upper right corner to show the localization of CD31 at cell-cell contacts. Length per structure and number of formed nodes was quantified using ImageJ. Analysis revealed a significantly higher structure length of mvECs on wet acdECM substrate compared to dry acdECM and stem cell approaches (dry and wet scdECM), comparable with FL approaches. Structure length on adipogenic FL was significantly higher compared with all approaches except wet acdECM. On adipogenic FL, a significantly higher number of nodes could be observed compared with all other approaches. acdECM, adipogenic cell-derived extracellular matrix; cdECM, cell-derived extracellular matrix; COL I, collagen I; FL, feeder layer; IF, immunofluorescence; mvECs, microvascular endothelial cells; scdECM, stem cell-derived extracellular matrix; TC, tissue culture. (Scale bar overview image: $200 \mu \mathrm{m}$; scale bar magnified section: $25 \mu \mathrm{m} ;{ }^{*} p \leq .05$;

${ }^{* *} p \leq .01 ;{ }^{* * *} p \leq .001$ ) [Color figure can be viewed at wileyonlinelibrary.com]

substrates was quantified. No nodes could be detected on the controls TC and COL I. In adipogenic approaches (dry acdECM: 1.2 [ \pm 1.1$]$; wet acdECM: $1.7[ \pm 1.2]$; and FL adipogenic: $6.7[ \pm 3.9]$ ) the number of nodes was higher compared with the stem cell approaches (dry scdECM: $0.7[ \pm 0.4]$; wet scdECM: $0.2[ \pm 0.6]$; and FL stem cell: 1.7 $[ \pm 1.5])$ for all substrates. Furthermore, the number of nodes on wet
acdECM was slightly but not significantly higher compared with the dry acdECM and comparable with stem cell FL. The significantly highest number of nodes could be observed in the adipogenic FL approach. To summarize, many short structures were identified on scdECM substrates, whereas on acdECM substrates the structures were longer and more branched. By coculture with the stem cell FL, 
mvECs formed islets of a confluent layer within the ASCs and prevascular-like structures sprouting from these islets were apparent. Long and highly branched prevascular-like structures were formed by mvECs on adipogenic FL.

\subsection{Quantification of proangiogenic factors on substrates}

To confine which $\mathrm{cdECM}$ components are responsible for its proangiogenic effect, the relative concentrations of growth factors VEGF, bFGF, and PDGF $\beta$ were determined in the supernatants after washing the acellular substrates for 3 days (Figure 5). Values were normalized to TC. The VEGF concentration was significantly higher for dry and wet acdECM substrates compared with all other acellular substrates. For quantification of growth factor concentrations on FL approaches, cell culture supernatant from Day 3 (corresponding to 3 days of washing of acellular substrates) was collected. A 10-fold higher concentration of VEGF could be found in FL approaches with no difference between stem cell FL and adipogenic FL significantly higher concentrations of bFGF could be found in cdECM substrates compared with controls and on FL approaches a higher concentration could be found compared with all other substrates. For PDGF $\beta$ a significantly higher concentration could be found on cdECM substrates compared with the controls. Between the different cdECM substrates no difference in PDGF $\beta$ concentration could be measured. On FL substrates, a higher concentration of PDGF $\beta$ could be found compared with acellular substrates but no difference between stem cell and adipogenically differentiated approach was observed.

\subsection{Expression of proteins associated with tube formation in newly formed prevascular-like structures}

Studies showed that the expression of adhesion molecules E-selectin and thrombomodulin in ECs is associated with the tube formation of new blood vessels (Oh et al., 2007; Pan et al., 2017). To get an indication if lumenogenesis occurs to any extent, we investigated the expression of these proteins in the newly formed prevascular-like structures (Figure 6). Results of the IF staining revealed the expression of neither E-selectin nor thrombomodulin in mvECs cultured on TC or COL I. However, all newly formed prevascular-like structures showed expression of E-selectin and thrombomodulin on all cdECM substrates. Furthermore, E-selectin and thrombomodulin expression of the prevascular-like structures were found in the newly formed prevascular-like structures on both FL approaches. E-selectin and thrombomodulin staining corresponded to the CD31 staining pattern of the prevascular-like structures (Figure S1).

\section{4 | DISCUSSION}

The implementation of a functional vascular system into an engineered tissue construct would address one of the major
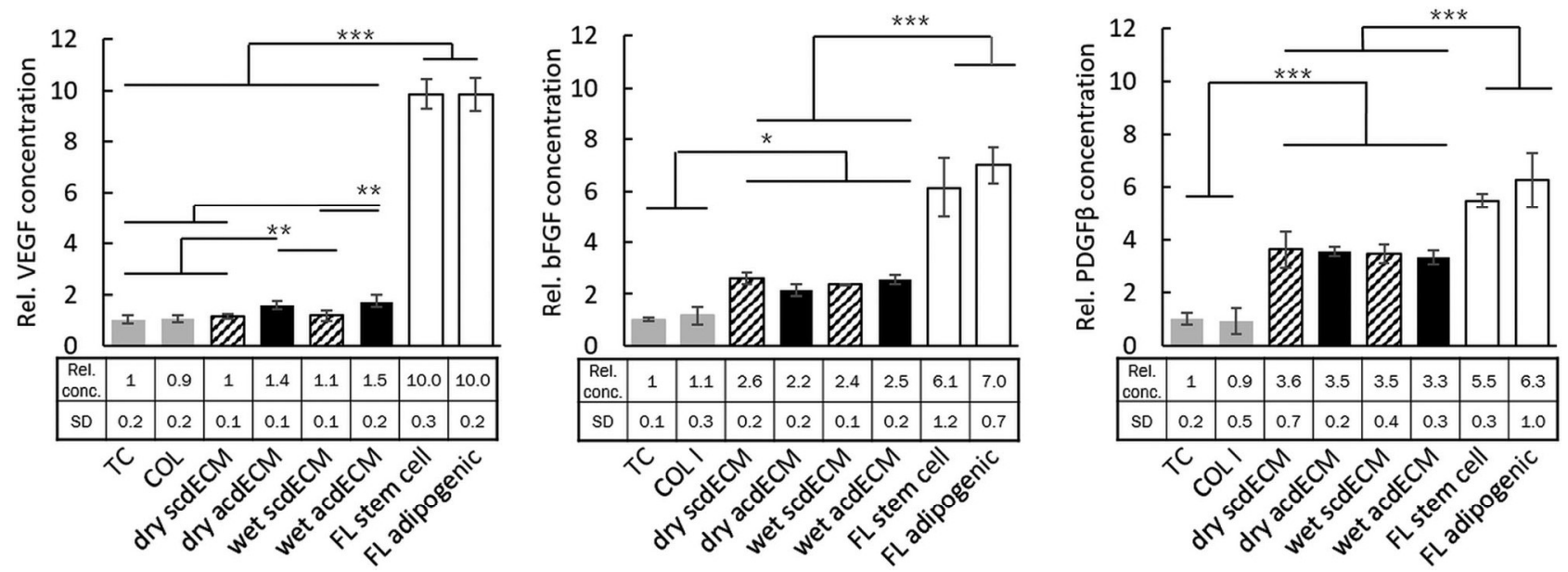

FIGURE 5 Proangiogenic factor concentrations on cellular and acellular ECM substrates. For the determination of VEGF, bFGF, and PDGF $\beta$ from the different substrates, supernatant from Day 3 was investigated regarding the concentration of the growth factors using ELISA. For statistical analysis, values on TC were set as 1 and data were normalized to TC. For VEGF, a significantly higher amount could be found in acdECM substrates (dry and wet) compared with all other acellular substrates including controls. FL substrates exhibited a 10-fold higher concentration of VEGF compared with acellular substrates. For bFGF, a higher concentration could be found in cdECM substrates compared with controls. On FL approaches, a higher concentration could be found compared with all other approaches. For PDGF $\beta$, a significantly higher (three-fold) amount could be found in all cdECM substrates compared with the controls TC and COL I. Between the individual cdECM substrates no difference in remaining PDGF $\beta$ could be found. FL substrates exhibited significantly higher PDGF $\beta$ concentrations compared with acellular substrates. acdECM, adipogenic cell-derived extracellular matrix; bFGF, basic fibroblast growth factor; cdECM, cell-derived extracellular matrix; COL I, collagen I; ELISA, enzyme-linked immunosorbent assay; FL, feeder layer; PDGF $\beta$, platelet-derived growth factor beta; scdECM, stem cell-derived extracellular matrix; TC, tissue culture; VEGF, vascular endothelial growth factor. $\left({ }^{*} p \leq .05 ;{ }^{* *} p \leq .01 ;{ }^{* * *} p \leq .001\right)$ 


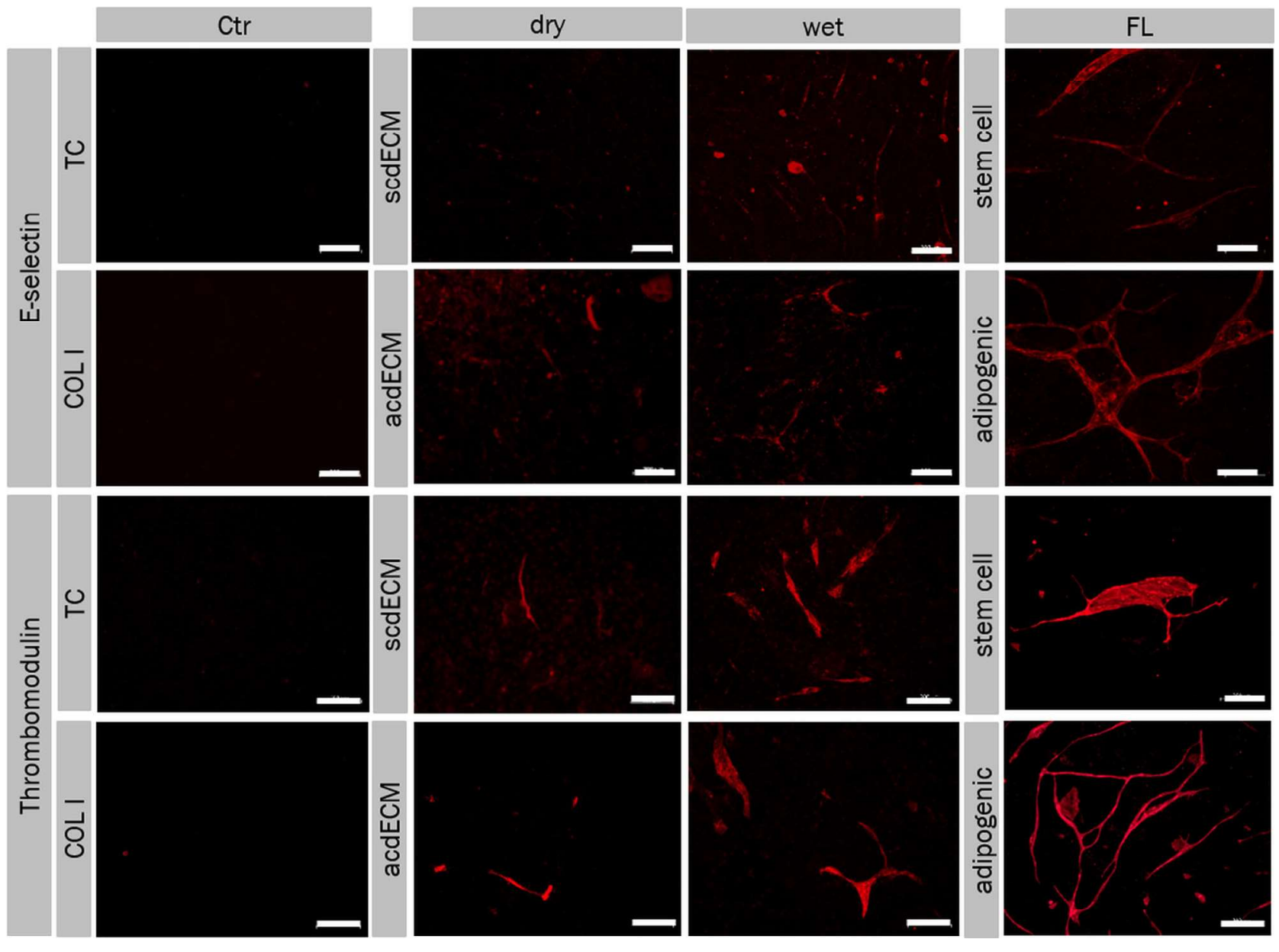

FIGURE 6 Expression of E-selectin and thrombomodulin by mvECs forming prevascular-like structures. About $1 \times 10^{4} \mathrm{mvECs} / \mathrm{cm}^{2}$ were seeded in defined coculture medium onto the different substrates and were cultured for 14 days. Medium was changed three times a week. For both proteins, no specific staining was observed on controls (TC and COL I coating). For all acellular and cellular substrates, specific staining of E-selectin and thrombomodulin (both indicated in red) could be found mainly on the newly formed prevascular-like structures. (Scale bar $=200 \mu \mathrm{m}$ ). acdECM: adipogenic cell-derived extracellular matrix; COL I, collagen I; FL, feeder layer; mvECs, microvascular endothelial cells; scdECM, stem cell-derived extracellular matrix; TC, tissue culture [Color figure can be viewed at wileyonlinelibrary.com]

bottlenecks in tissue engineering and regenerative medicine. In the present study, we aimed to investigate the supportive effect of cdECM on the self-assembled formation of prevascular-like structures by mvECs for its use as a biomaterial for adipose tissue engineering compared with the well-established application of a supportive FL.

Determination of the degree of swelling revealed a higher capacity of water uptake of the adipogenic ECM compared with stem cell ECM. This effect can be explained by the larger pore size in acdECM shown by fibronectin staining. The development of larger pores in acdECM may be attributed to morphological changes of ASCs during adipogenesis. By incorporating lipids, adipogenically differentiated ASCs develop a more spherical shape compared with the stem cells and substantially increase their volume (Moldovan et al., 2019). During adipogenesis, ASCs further stop to proliferate which results in a lower total cell number in adipogenic approaches (Fajas, 2003). Thus, the amount of cdECM in stem cell approaches may be higher which leads to more densely packed collagen fibers and smaller pores. The larger pores found in adipogenic ECM might also be able to enhance the degree of prevascular-like structure formation by mvECs. Chui et al. (2011) showed that larger pore size is associated with a higher degree of neovascularization in an in vitro PEG hydrogel model. Furthermore, Artel, Mehdizadeh, Chiu, Brey, and Cinar (2011) proposed an agent-based model indicating that pores of larger size support vascularization in a polymer scaffold.

Analysis of LDH release of mvECs on the substrates revealed no cytotoxic effect of the cdECM substrates, the FL cells or the controls (COL I coating and TC). Even on Day 14 after seeding, a confluent viable monolayer of mvECs was observed which indicates a good cytocompatibility of the cdECM substrates and their possible use in tissue engineering. Nearly no dead mvECs were observed during the quantification of the live/dead staining on acellular substrates. For the stem cell FL approach, $<1 \%$ of dead cells could be found. However, on the adipogenic FL approach around $4 \%$ of dead cells could be found for FL with and without mvECs. Adipogenically differentiated ASCs may be more sensitive to the change to the defined coculture 
medium compared with the nondifferentiated ASCs which leads to an increase of cell death. For comprehensive toxicological and immunogenic characterization further analysis is required, for example, the analysis of the cdECM impact on the metabolic activity of the mvECs and for the intended in vivo use, biocompatibility of the CdECM has to be evaluated.

Visualization of mvECs on Day 14 after seeding by staining of the specific surface protein CD31 showed the self-assembled formation of prevascular-like structures on all substrates except for the controls COL I and TC. Structure formation on the adipogenic FL approach was in line with our previous study (Volz et al., 2018) as a lower degree of structure formation was found on the stem cell FL approach. In addition, on dry and wet cdECM approaches, the degree of prevascular-like structure formation on adipogenic ECM substrates was higher compared with the corresponding stem cell approach, which is reflected by longer structures and a higher number of nodes. The effect of enhanced structure formation on adipogenic substrates could be explained by the different secretomes of ASCs and (pre-)adipocytes (Kapur \& Katz, 2013). It is well known that ASCs secrete a broad spectrum of proangiogenic proteins and they were often used as a delivery system of growth factors and cytokines in vascularization approaches (Kondo et al., 2009; Liu et al., 2011; Moon et al., 2006; Nakagami, Maeda, Kaneda, Ogihara, \& Morishita, 2005; Rehman et al., 2004). For example, Matsuda et al. (2013) showed that conditioned cell culture medium of ASCs positively influenced EC proliferation and the formation of new vessels in vivo. During adipogenic differentiation, ASCs secret further proangiogenic factors like leptin. Leptin is known to be upregulated during adipogenic differentiation and was shown to exhibit a proangiogenic effect itself but also upregulates the secretion of VEGF (Cao, Brakenhielm, Wahlestedt, Thyberg, \& Cao, 2001). By secreting their specific set of proteins, ASCs and (pre-)adipocytes not only condition their cell culture medium but also their ECM which we use in this study as a biomaterial for induction of prevascular-like structure formation by mvECs. Thus, cdECM does not only contain a set of specific factors, but a broad spectrum of proangiogenic factors with its synergistic effects needed for the successful formation of prevascular-like structures by ECs. Especially acdECM supports the formation of prevascular-like structures and seems to be able to stabilize the newly formed structures.

The two most important proangiogenic factors are VEGF and bFGF. Results revealed higher VEGF concentrations released from acdECM substrates compared with scdECM approaches. On FL approaches, high amounts of VEGF were found, most likely produced by FL cells. These results are in line with the degree of prevascular-like structure formation. On acdECM approaches, longer and more branched structures were formed whereas on $\mathrm{FL}$ approaches the highest degree of structure formation occurred. Determination of the bFGF concentration in the different substrates revealed a higher concentration from cdECM substrates compared with controls and the highest bFGF concentration from FL approaches. Both factors, VEGF and bFGF, are able to induce the formation of new vascular structures (Marra et al., 2008; Murakami \& Simons, 2008; Nissen et al., 2007; Tomanek, Hansen, \& Christensen, 2008). Therefore, in our study, the induction of the formation of prevascular-like structures might among other events, be attributed to the synergistic effect of available VEGF and bFGF. We further investigated the amount of the proangiogenic factor PDGF $\beta$ from cdECM substrates. It is secreted by ECs during angiogenesis to attract perivascular cells, which stabilize the newly formed vessels (Gaengel et al., 2009). Furthermore, PDGF $\beta$ was shown to induce vascular structure formation by modulating proliferation and tube formation of ECs (Battegay, Rupp, Iruela-Arispe, Sage, \& Pech, 1994). PDGF $\beta$ can be found in all cdECM substrates as well as FL approaches. The PDGF $\beta$ concentration from FL approaches is higher compared with the other substrates, which is in line with the higher degree of structure formation. In vivo, these growth factors are known to be partially bound to ECM after their secretion (Ostman, Andersson, Betsholtz, Westermark, \& Heldin, 1991). To date, there are no studies investigating their binding capacity and protein half-life in in vitro generated cdECM.

A critical step in the formation of a new vascular system is the formation of a lumen in the vascular structure to enable perfusion with blood in vivo and culture medium in vitro. Studies show that the adhesion proteins E-selectin and thrombomodulin are associated with tube formation. In vivo, E-selectin is mainly contributing to the binding of immune cells by mediating adhesive interactions of circulating leukocytes with the endothelium (Ley \& Tedder, 1995). Nevertheless, it also plays a role in the homing of endothelial progenitor cells (EPCS) and therefore promotes neovascularization. Studies showed that E-selectin potentiates angiogenesis in ischemic tissue, by mediating EPC-endothelial interactions (Oh et al., 2007). During this process of neovascularization, EPCs are mobilized from the bone marrow into the circulation and recruited to new sites of vascularization, using cues that resemble an inflammatory response. Therefore, E-selectin plays a crucial role in EPC homing and following neovascularization and tube formation. Thrombomodulin is a transmembrane protein expressed on ECs acting as an anticoagulant (Dahlback \& Villoutreix, 2005; Dittman \& Majerus, 1990). The fourth and fifth region of an EGF-like region of thrombomodulin (TME45) was shown to stimulate the proliferation of human umbilical vein ECs and to promote tube formation and angiogenesis (Ikezoe et al., 2017). In this study, we use these proteins as indicators for the development of the prevascular-like structure towards a tubular vascular structure with a lumen. IF staining of E-selectin and thrombomodulin revealed specific expression of E- selectin almost exclusively on the newly formed prevascular-like structures. Thus, we suggest that newly formed prevascular-like structures exhibit promising characteristics to develop a lumen. The expression of E-selectin and thrombomodulin and their function in tube formation and neovascularization in vivo represent promising characteristics when considering implantation of prevascularized constructs.

Compared with ECM derived from native tissue, cdECM exhibited a variety of advantages, which confirms it as a promising biomaterial for tissue engineering. For example, the possibility of autologous production without harvesting high amounts of 
autologous tissue and the generation of ECM from different developmental stages. This study confirms a supportive effect of cdECM on the spontaneous formation of prevascular-like structures by mvECs. Furthermore, it could be shown that dry cdECM partly maintains its biological properties regarding the induction of selfassembled prevascular-like structure formation of mvECs with some restrictions. Drying of the $\mathrm{CdECM}$ would be a convenient method to improve storage possibilities when necessary. Due to the relatively low amounts of cdECM, which can be produced with current methods, this study is limited to $2 \mathrm{D}$ approaches, which insufficiently reflect physiological conditions. Further studies should focus on the upscaling of the generation of cdECM to enable the setup of continuative experiments in $3 \mathrm{D}$ constructs consisting of $\mathrm{cdECM}$, which would better reflect the situation in vivo.

\section{5 | CONCLUSION}

In the present study, we demonstrated that CdECM (as a dry coating and as a wet hydrogel-like form) is able to induce the self-assembled formation of prevascular-like structures by mvECs and helps to support their maintenance. Mainly acdECM was confirmed as a promising material for adipose tissue engineering by supporting the formation of prevascular-like structures. In addition, scdECM also provides the ability to induce prevascular-like structure formation and can be used for approaches addressing other tissues. In future investigations regarding other lineage-specific cdECMs, the upscaling of cdECM generation and the transfer from 2D cell culture to 3D cell culture should be pursued.

\section{ACKNOWLEDGMENTS}

This study was financially supported by the Landesgraduiertenförderung by the Ministry of Science, Research, and the Arts (Baden-Württemberg, Germany) under the program "Intelligent Process and Material Development in Biomateriomics" (University of Tuebingen and Reutlingen University).

\section{CONFLICT OF INTERESTS}

The authors declare that there are no conflict of interests.

\section{ORCID}

Svenja Nellinger (D) http://orcid.org/0000-0001-7281-2196

\section{REFERENCES}

Adam Young, D., Bajaj, V., \& Christman, K. L. (2014). Award winner for outstanding research in the PhD category, 2014 Society for Biomaterials annual meeting and exposition, Denver, Colorado, April 16-19, 2014: Decellularized adipose matrix hydrogels stimulate in vivo neovascularization and adipose formation. Journal of Biomedical Materials Research. Part A, 102(6), 1641-1651. https://doi.org/10. 1002/jbm.a.35109

Artel, A., Mehdizadeh, H., Chiu, Y. C., Brey, E. M., \& Cinar, A. (2011). An agent-based model for the investigation of neovascularization within porous scaffolds. Tissue Engineering. Part A, 17(17-18), 2133-2141. https://doi.org/10.1089/ten.TEA.2010.0571
Badylak, S. F., Freytes, D. O., \& Gilbert, T. W. (2009). Extracellular matrix as a biological scaffold material: Structure and function. Acta Biomaterialia, 5(1), 1-13. https://doi.org/10.1016/j.actbio.2008.09.013

Battegay, E. J., Rupp, J., Iruela-Arispe, L., Sage, E. H., \& Pech, M. (1994). PDGF-BB modulates endothelial proliferation and angiogenesis in vitro via PDGF beta-receptors. Journal of Cell Biology, 125(4), 917-928. https://doi.org/10.1083/jcb.125.4.917

Bayless, K. J., \& Davis, G. E. (2002). The Cdc42 and Rac1 GTPases are required for capillary lumen formation in three-dimensional extracellular matrices. Journal of Cell Science, 115(Pt 6), 1123-1136.

Cao, R., Brakenhielm, E., Wahlestedt, C., Thyberg, J., \& Cao, Y. (2001). Leptin induces vascular permeability and synergistically stimulates angiogenesis with FGF-2 and VEGF. Proceedings of the National Academy of Sciences of the United States of America, 98(11), 6390-6395. https://doi.org/10.1073/pnas.101564798

Chiu, Y. C., Cheng, M. H., Engel, H., Kao, S. W., Larson, J. C., Gupta, S., \& Brey, E. M. (2011). The role of pore size on vascularization and tissue remodeling in PEG hydrogels. Biomaterials, 32(26), 6045-6051. https://doi.org/10.1016/j.biomaterials.2011.04.066

Dahlback, B., \& Villoutreix, B. O. (2005). The anticoagulant protein C pathway. FEBS Letters, 579(15), 3310-3316. https://doi.org/10.1016/j. febslet.2005.03.001

Dittman, W. A., \& Majerus, P. W. (1990). Structure and function of thrombomodulin: A natural anticoagulant. Blood, 75(2), 329-336.

Dzobo, K., Turnley, T., Wishart, A., Rowe, A., Kallmeyer, K., van Vollenstee, F. A., ... Parker, M. I. (2016). Fibroblast-derived extracellular matrix induces chondrogenic differentiation in human adipose-derived mesenchymal stromal/stem cells in vitro. International Journal of Molecular Sciences, 17(8), https://doi.org/10.3390/ ljms17081259

Fajas, L. (2003). Adipogenesis: A cross-talk between cell proliferation and cell differentiation. Annals of Medicine, 35(2), 79-85. https://doi.org/ 10.1080/07853890310009999

Flynn, L., Prestwich, G. D., Semple, J. L., \& Woodhouse, K. A. (2009). Adipose tissue engineering in vivo with adipose-derived stem cells on naturally derived scaffolds. Journal of Biomedical Materials Research. Part A, 89A(4), 929-941. https://doi.org/10.1002/jbm.a.32044

Flynn, L. E. (2010). The use of decellularized adipose tissue to provide an inductive microenvironment for the adipogenic differentiation of human adipose-derived stem cells. Biomaterials, 31(17), 4715-4724. https://doi.org/10.1016/j.biomaterials.2010.02.046

Gaengel, K., Genove, G., Armulik, A., \& Betsholtz, C. (2009). Endothelialmural cell signaling in vascular development and angiogenesis. Arteriosclerosis, Thrombosis, and Vascular Biology, 29(5), 630-638. https://doi.org/10.1161/ATVBAHA.107.161521

Guneta, V., Loh, Q. L., \& Choong, C. (2016). Cell-secreted extracellular matrix formation and differentiation of adipose-derived stem cells in 3D alginate scaffolds with tunable properties. Journal of Biomedical Materials Research. Part A, 104(5), 1090-1101. https://doi.org/10. 1002/jbm.a.35644

Guneta, V., Zhou, Z., Tan, N. S., Sugii, S., Wong, M. T. C., \& Choong, C. (2017). Recellularization of decellularized adipose tissue-derived stem cells: Role of the cell-secreted extracellular matrix in cellular differentiation. Biomaterials Science, 6(1), 168-178. https://doi.org/ 10.1039/c7bm00695k

Guo, Y., Zeng, Q. C., Yan, Y. X., Shen, L., Liu, L., Li, R. X., ... Huang, S. J. (2013). Proliferative effect and osteoinductive potential of extracellular matrix coated on cell culture plates. SpringerPlus, 2, 303. https://doi.org/10.1186/2193-1801-2-303

Huber, B., Borchers, K., Tovar, G. E., \& Kluger, P. J. (2016). Methacrylated gelatin and mature adipocytes are promising components for adipose tissue engineering. Journal of Biomaterials Applications, 30(6), 699-710. https://doi.org/10.1177/0885328215587450

Ikezoe, T., Yang, J., Nishioka, C., Pan, B., Xu, K., Furihata, M., ... Yokoyama, A. (2017). The fifth epidermal growth factor-like region of 
thrombomodulin exerts cytoprotective function and prevents SOS in a murine model. Bone Marrow Transplantation, 52(1), 73-79. https:// doi.org/10.1038/bmt.2016.195

Kapur, S. K., \& Katz, A. J. (2013). Review of the adipose derived stem cell secretome. Biochimie, 95(12), 2222-2228. https://doi.org/10.1016/j. biochi.2013.06.001

Kondo, K., Shintani, S., Shibata, R., Murakami, H., Murakami, R. Imaizumi, M., ... Murohara, T. (2009). Implantation of adipose-derived regenerative cells enhances ischemia-induced angiogenesis. Arteriosclerosis, Thrombosis, and Vascular Biology, 29(1), 61-U167. https://doi.org/10.1161/Atvbaha.108.166496

Laschke, M. W., \& Menger, M. D. (2016). Prevascularization in tissue engineering: Current concepts and future directions. Biotechnology Advances, 34(2), 112-121. https://doi.org/10.1016/j.biotechadv.2015. 12.004

Laschke, M. W., Rucker, M., Jensen, G., Carvalho, C., Mulhaupt, R., Gellrich, N. C., \& Menger, M. D. (2008). Incorporation of growth factor containing Matrigel promotes vascularization of porous PLGA scaffolds. Journal of Biomedical Materials Research. Part A, 85(2), 397-407. https://doi.org/10.1002/jbm.a.31503

Laschke, M. W., Strohe, A., Scheuer, C., Eglin, D., Verrier, S., Alini, M., ... Menger, M. D. (2009). In vivo biocompatibility and vascularization of biodegradable porous polyurethane scaffolds for tissue engineering. Acta Biomaterialia, 5(6), 1991-2001. https://doi.org/10.1016/j.actbio. 2009.02.006

Laschke, M. W., Vollmar, B., \& Menger, M. D. (2009). Inosculation: Connecting the life-sustaining pipelines. Tissue Engineering. Part B, Reviews, 15(4), 455-465. https://doi.org/10.1089/ten.TEB.2009.0252

Ley, K., \& Tedder, T. F. (1995). Leukocyte interactions with vascular endothelium. New insights into selectin-mediated attachment and rolling. Journal of Immunology, 155(2), 525-528.

Liu, S., Zhang, H., Zhang, X., Lu, W., Huang, X., Xie, H., ... Jin, Y. (2011). Synergistic angiogenesis promoting effects of extracellular matrix scaffolds and adipose-derived stem cells during wound repair. Tissue Engineering. Part A, 17(5-6), 725-739. https://doi.org/10.1089/ten. TEA.2010.0331

Lu, H. X., Hoshiba, T., Kawazoe, N., \& Chen, G. P. (2011). Autologous extracellular matrix scaffolds for tissue engineering. Biomaterials, 32(10), 2489-2499. https://doi.org/10.1016/j.biomaterials.2010.12.016

Lu, H. X., Hoshiba, T., Kawazoe, N., Koda, I., Song, M. H., \& Chen, G. P. (2011). Cultured cell-derived extracellular matrix scaffolds for tissue engineering. Biomaterials, 32(36), 9658-9666. https://doi.org/10. 1016/j.biomaterials.2011.08.091

Marra, K. G., DeFail, A., Clavijo-Alvarez, J. A., Badylak, S. F., Taieb, A. Schipper, B., ... Rubin, J. P. (2008). FGF-2 enhances vascularization for adipose tissue engineering. Plastic and Reconstructive Surgery, 121(4), 1153-1164. https://doi.org/10.1097/01.prs.0000305517.93747.72

Matsuda, K., Falkenberg, K. J., Woods, A. A., Choi, Y. S., Morrison, W. A., \& Dilley, R. J. (2013). Adipose-derived stem cells promote angiogenesis and tissue formation for in vivo tissue engineering. Tissue Engineering. Part A, 19(11-12), 1327-1335. https://doi.org/10.1089/ten.TEA. 2012.0391

Moldovan, L. M. Y., Lustig, M., Naftaly, A., Mardamshina, M., Geiger, T., Gefen, A., \& Benayahu, D. (2019). Cell shape alteration during adipogenesis is associated with coordinated matrix cues. Journal of Cellular Physiology, 234(4), 3850-3863. https://doi.org/10.1002/jcp. 27157

Moon, M. H., Kim, S. Y., Kim, Y. J., Kim, S. J., Lee, J. B., Bae, Y. C., ... Jung, J. S. (2006). Human adipose tissue-derived mesenchymal stem cells improve postnatal neovascularization in a mouse model of hindlimb ischemia. Cellular Physiology and Biochemistry, 17(5-6), 279-290. https://doi.org/10.1159/000094140

Murakami, M., \& Simons, M. (2008). Fibroblast growth factor regulation of neovascularization. Current Opinions in Hematology, 15(3), 215-220. https://doi.org/10.1097/MOH.0b013e3282f97d98
Nakagami, H., Maeda, K., Kaneda, Y., Ogihara, T., \& Morishita, R. (2005). Novel autologous cell therapy in ischemic limb disease through growth factor secretion by cultured adipose tissue-derived stromal cells. Hypertension, 46(4), 867.

Nissen, L. J., Cao, R., Hedlund, E. M., Wang, Z., Zhao, X., Wetterskog, D., ... Cao, Y. (2007). Angiogenic factors FGF2 and PDGF-BB synergistically promote murine tumor neovascularization and metastasis. Journal of Clinical Investigation, 117(10), 2766-2777. https://doi.org/10.1172/ $\mathrm{JCl} 32479$

Oh, I. Y., Yoon, C. H., Hur, J., Kim, J. H., Kim, T. Y., Lee, C. S., ... Kim, H. S. (2007). Involvement of E-selectin in recruitment of endothelial progenitor cells and angiogenesis in ischemic muscle. Blood, 110(12) 3891-3899. https://doi.org/10.1182/blood-2006-10-048991

Olive, P. L., Vikse, C., \& Trotter, M. J. (1992). Measurement of oxygen diffusion distance in tumor cubes using a fluorescent hypoxia probe. International Journal of Radiation Oncology, Biology, Physics, 22(3), 397-402. https://doi.org/10.1016/0360-3016(92)90840-E

Ostman, A., Andersson, M., Betsholtz, C., Westermark, B., \& Heldin, C. H. (1991). Identification of a cell retention signal in the B-chain of platelet-derived growth factor and in the long splice version of the Achain. Cell Regulation, 2(7), 503-512. https://doi.org/10.1091/mbc.2 7.503

Pan, B., Wang, X., Nishioka, C., Honda, G., Yokoyama, A., Zeng, L., .. Ikezoe, T. (2017). G-protein coupled receptor 15 mediates angiogenesis and cytoprotective function of thrombomodulin. Scientific Reports, 7(1), 692. https://doi.org/10.1038/s41598-017 00781-w

Piali, L., Hammel, P., Uherek, C., Bachmann, F., Gisler, R. H., Dunon, D., \& Imhof, B. A. (1995). CD31/PECAM-1 is a ligand for alpha $\vee$ beta 3 integrin involved in adhesion of leukocytes to endothelium. Journal of Cell Biology, 130(2), 451-460. https://doi.org/10.1083/jcb.130.2.451

Rehman, J., Traktuev, D., Li, J., Merfeld-Clauss, S., Temm-Grove, C. J. Bovenkerk, J. E., ... March, K. L. (2004). Secretion of angiogenic and antiapoptotic factors by human adipose stromal cells. Circulation, 109(10), 1292-1298. https://doi.org/10.1161/01.CIR.0000121425. 42966.F1

Sart, S., Yan, Y. W., Li, Y., Lochner, E., Zeng, C. C., Ma, T., \& Li, Y. (2016) Crosslinking of extracellular matrix scaffolds derived from pluripotent stem cell aggregates modulates neural differentiation. Acto Biomaterialia, 30, 222-232. https://doi.org/10.1016/j.actbio.2015. 11.016

Schenke-Layland, K., Rofail, F., Heydarkhan, S., Gluck, J. M., Ingle, N. P., Angelis, E., ... Heydarkhan-Hagvall, S. (2009). The use of threedimensional nanostructures to instruct cells to produce extracellular matrix for regenerative medicine strategies. Biomaterials, 30(27), 4665-4675. https://doi.org/10.1016/j.biomaterials.2009.05.033

Thomlinson, R. H., \& Gray, L. H. (1955). The histological structure of some human lung cancers and the possible implications for radiotherapy. British Journal of Cancer, 9(4), 539-549. https://doi.org/10.1038/bjc. 1955.55

Tomanek, R. J., Hansen, H. K., \& Christensen, L. P. (2008). Temporally expressed PDGF and FGF-2 regulate embryonic coronary artery formation and growth. Arteriosclerosis, Thrombosis, and Vascular Biology, 28(7), 1237-1243. https://doi.org/10.1161/ATVBAHA.108. 166454

Verseijden, F., Posthumus-van Sluijs, S. J., Farrell, E., van Neck, J. W., Hovius, S. E. R., Hofer, S. O. P., \& van Osch, G. J. V. M. (2010) Prevascular structures promote vascularization in engineered human adipose tissue constructs upon implantation. Cell Transplantation 19(8), 1007-1020. https://doi.org/10.3727/096368910X492571

Verseijden, F., Posthumus-van Sluijs, S. J., van Neck, J. W., Hofer, S. O., Hovius, S. E., \& van Osch, G. J. (2012). Comparing scaffold-free and fibrin-based adipose-derived stromal cell constructs for adipose tissue engineering: An in vitro and in vivo study. Cell Transplantation, 21(10), 2283-2297. https://doi.org/10.3727/096368912X653129 
Verseijden, F., Posthumus-van Sluijs, S. J., Pavljasevic, P., Hofer, S. O. P., van Osch, G. J. V. M., \& Farrell, E. (2010). Adult human bone marrow- and adipose tissue-derived stromal cells support the formation of prevascularlike structures from endothelial cells in vitro. Tissue Engineering. Part A, 16(1), 101-114. https://doi.org/10.1089/ten.tea.2009.0106

Volz, A. C., Hack, L., Atzinger, F. B., \& Kluger, P. J. (2018). Completely defined co-culture of adipogenic differentiated ASCs and microvascular endothelial cells. Altex-Alternatives to Animal Experimentation, 35(4), 464-476. https://doi.org/10.14573/altex.1802191

Volz, A. C., Huber, B., Schwandt, A. M., \& Kluger, P. J. (2017). EGF and hydrocortisone as critical factors for the co-culture of adipogenic differentiated ASCs and endothelial cells. Differentiation, 95, 21-30. https://doi.org/10.1016/j.diff.2017.01.002

Walser, R., Metzger, W., Gorg, A., Pohlemann, T., Menger, M. D., \& Laschke, M. W. (2013). Generation of co-culture spheroids as vascularisation units for bone tissue engineering. European Cells \& Materials, 26, 222-233. https://doi.org/10.22203/ecm.v026a16

Wenz, A., Tjoeng, I., Schneider, I., Kluger, P. J., \& Borchers, K. (2018). Improved vasculogenesis and bone matrix formation through coculture of endothelial cells and stem cells in tissue-specific methacryloyl gelatinbased hydrogels. Biotechnology and Bioengineering, 115(10), 2643-2653. https://doi.org/10.1002/bit.26792

Wolchok, J. C., \& Tresco, P. A. (2010). The isolation of cell derived extracellular matrix constructs using sacrificial open-cell foams.
Biomaterials, 31(36), 9595-9603. https://doi.org/10.1016/j. biomaterials.2010.08.072

Yoon, J. J., Chung, H. J., Lee, H. J., \& Park, T. G. (2006). Heparinimmobilized biodegradable scaffolds for local and sustained release of angiogenic growth factor. Journal of Biomedical Materials Research. Part A, 79A(4), 934-942. https://doi.org/10.1002/jbm.a.30843

\section{SUPPORTING INFORMATION}

Additional supporting information may be found online in the Supporting Information section.

How to cite this article: Nellinger S, Schmidt I, Heine S, Volz A-C, Kluger PJ. Adipose stem cell-derived extracellular matrix represents a promising biomaterial by inducing spontaneous formation of prevascular-like structures by mvECs.

Biotechnology and Bioengineering. 2020;117:3160-3172.

https://doi.org/10.1002/bit.27481 